\title{
Clues from microquasars to the origin of radio-loudness of quasars
}

\author{
Carlo Nipoti* \\ Università di Bologna, Italy \\ E-mail: carlo.nipoti@unibo.it
}

\section{Katherine M. Blundell}

University of Oxford, Department of Physics, Keble Road, Oxford, OXI 3RH, U.K.

\section{James Binney}

University of Oxford, Department of Physics, Keble Road, Oxford, OX1 3RH, U.K.

\begin{abstract}
We analysed the long-term variability of four microquasars (GRS 1915+105, Cyg X-1, Cyg X-3, and Sco X-1) in radio and X rays. The results of our analysis indicate the existence of two distinct modes of energy output, which we refer to as the 'coupled' mode and the 'flaring' mode. The coupled mode is responsible for mildly fluctuating, flat-spectrum radio emission, coupled with the X-ray emission; the flaring mode produces powerful, steep-spectrum radio flares, with no significant counterpart in $\mathrm{X}$ rays. We find that the fraction of time spent by a typical microquasar in the flaring mode is similar to the fraction of quasars that are radio-loud. This is consistent with the hypothesis that radio-loudness of quasars is a function of the epoch at which the source is observed.
\end{abstract}

VI Microquasar Workshop: Microquasars and Beyond September 18-22, 2006

Como, Italy

\footnotetext{
* Speaker.
} 


\section{Introduction}

Microquasars are powered by accreting stellar mass black holes or neutron stars, so they can be considered scale models of active galactic nuclei (AGN). The characteristic times for the evolution of these sources scale with the mass of the accreting object, so long-term monitoring of microquasars represents a unique opportunity to get insights on the variability properties of quasars over timescales up to $10^{8}-10^{9} \mathrm{yr}$. We exploit this scaling between microquasars and AGN to obtain clues to the long-standing question of the origin of radio-loudness of quasars. In particular, we explore the possibility that many quasars that are now radio-quiet may be at times radio-loud, while currently radio-loud quasars may often be radio-quiet.

We analysed the long-term variability in radio and in X-rays of the microquasars GRS 1915+105, Cyg X-3, Sco X-1 and Cyg X-1. We used data from the archive of the US National Radio Astronomy Observatory Green Bank Interferometer ${ }^{1}$ (at $2.25 \mathrm{GHz}$ and $8.3 \mathrm{GHz}$ ) and the Rossi X-ray Timing Explorer All-Sky Monitor ${ }^{2}$ (at 2-10 keV). In particular, we considered daily-averaged Xray and radio light-curves. The X-ray light-curves of the four sources refer to the period $50087-$ 53300 (J.D.-2400000); the periods covered by the radio light curves differ from source to source (49485 - 51823 for GRS 1915+105, 44915 - 51823 for Cyg X-3, 50638 - 51823 for Sco X-1, 50412 - 51823 for Cyg X-1; J.D.-2400000). Here we briefly report some results we presented and discussed in detail in [1], together with new results on the variability of the radio spectra of these sources.

\section{Long-term variability of microquasars in radio and in X-rays}

Figure 1 plots the power spectra of the four considered microquasars in radio (at $2.25 \mathrm{GHz}$; red symbols) and X-rays (blue symbols), in units of $(\mathrm{rms} /\langle\mathrm{L}\rangle)^{2} \mathrm{~Hz}^{-1}$, where $\langle L\rangle$ is the average luminosity. It is apparent that the radio and X-ray power spectra of each source have similar shape, but, while in the case of Cyg X-1 their normalisations are also similar, for the other three sources the radio power spectra are higher (by factors up to $\sim 30$ ) than the corresponding X-ray power spectra. This reflects the fact that, while the X-ray luminosity never exceeds three times its median value, the radio luminosity is above this threshold for a significant fraction of the time in GRS 1915+105, Cyg X-3, and Sco X-1, with peaks up to two orders of magnitude above the median. On the other hand, in Cyg X-1 the radio luminosity behaves in this respect as the X-ray luminosity, being always below three times the median. These results suggest that two distinct modes contribute to radio activity: the 'coupled' mode, producing also X-ray emission, and the 'flaring' mode, producing strong radio flares, but no corresponding strong X-ray emission. This interpretation is supported by the diagrams in Fig. 2, showing, for daily-averaged data points of the four sources, the radio spectral index $\alpha_{2-8 \mathrm{~Hz}} \equiv \log \left(L_{8 \mathrm{GHz}} / L_{2 \mathrm{GHz}}\right) / \log (8.3 / 2.25)$ as a function of $L_{2 \mathrm{GHz}}$ in units of its median value $L_{2 \mathrm{GHz} \text {,med }}$ (where $L_{8 \mathrm{GHz}}$ and $L_{2 \mathrm{GHz}}$ are the $8.3 \mathrm{GHz}$ and $2.25 \mathrm{GHz}$ luminosities, respectively). In the case of GRS $1915+105$, Cyg X-3 and Sco X-1 there is an apparent segregation of the data points in these diagrams: in the flaring mode (i.e., when $L_{2 \mathrm{GHz}} \gtrsim 3 L_{2 \mathrm{GHz} \text {,med }}$ ) the radio

\footnotetext{
${ }^{1}$ http://www.gb.nrao.edu/fgdocs/gbi/gbint.html

${ }^{2}$ http://xte.mit.edu/ASM_lc.html
} 


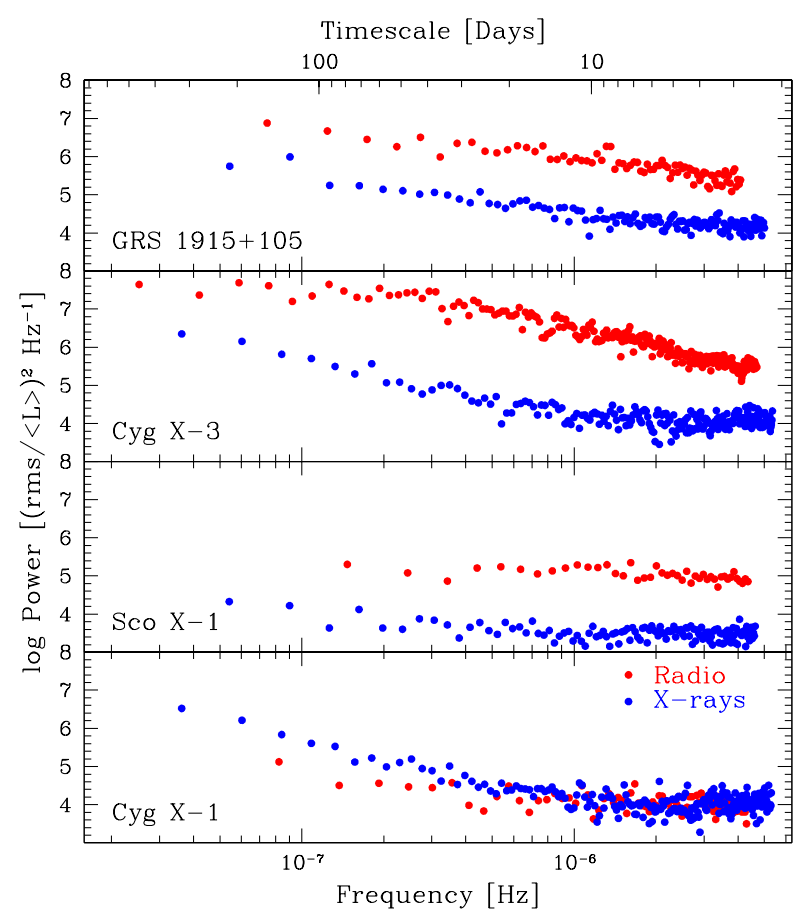

Figure 1: Radio (2.25 GHz; red symbols) and X-ray (2-10 keV; blue symbols) power spectra of GRS 1915+105, Cyg X-3, Sco X-1 and Cyg X-1.

spectrum is typically steeper than in the coupled mode $\left(L_{2 \mathrm{GHz}} \lesssim 3 L_{2 \mathrm{GHz} \text {,med }}\right)$. Consistently, Cyg X1 , which has not been observed flaring during the radio monitoring period, always has a flat radio spectrum. GRS 1915+105, Cyg X-3, Sco X-1 are in the flaring mode 21, 10, and 3 per cent of the time, respectively. In terms of the canonical X-ray states of microquasars, the coupled mode is associated with both the high/soft and the low/hard states, while the flaring mode occurs at the transition between these two states, in the so-called intermediate X-ray state. Both the coupled and flaring mechanisms are active in the neutron-star powered source Sco X-1, so neither mechanism is associated with the extraction of energy from black-hole spin. However, we note that Sco X-1 presents some difference with respect to the three black-hole powered sources: it is less variable in X-rays (see Fig. 1) - probably because of the contribution to the X-ray luminosity from the surface of the neutron star- and tends to have steeper radio spectrum also in the coupled mode (see Fig. 2).

\section{Implications for the origin of radio-loudness of quasars}

Long-term radio and X-ray monitoring of microquasars indicates that these sources, during their lifetime, often switch between a radio-loud (flaring) and a radio-quiet (coupled) mode. If AGN behave similarly, radio-loudness of quasars would be just a function of the epoch at which the source is observed. It must be noted that the radio luminosity produced in the flaring mode is expected to be proportionally smaller in microquasars than in radio galaxies because the ambient medium around microquasars may not be dense enough to produce bright lobes ([2]). However, with an appropriate choice of the threshold for radio-loudness (see [1]), the fraction of time $f_{\mu \mathrm{Q}}$ 


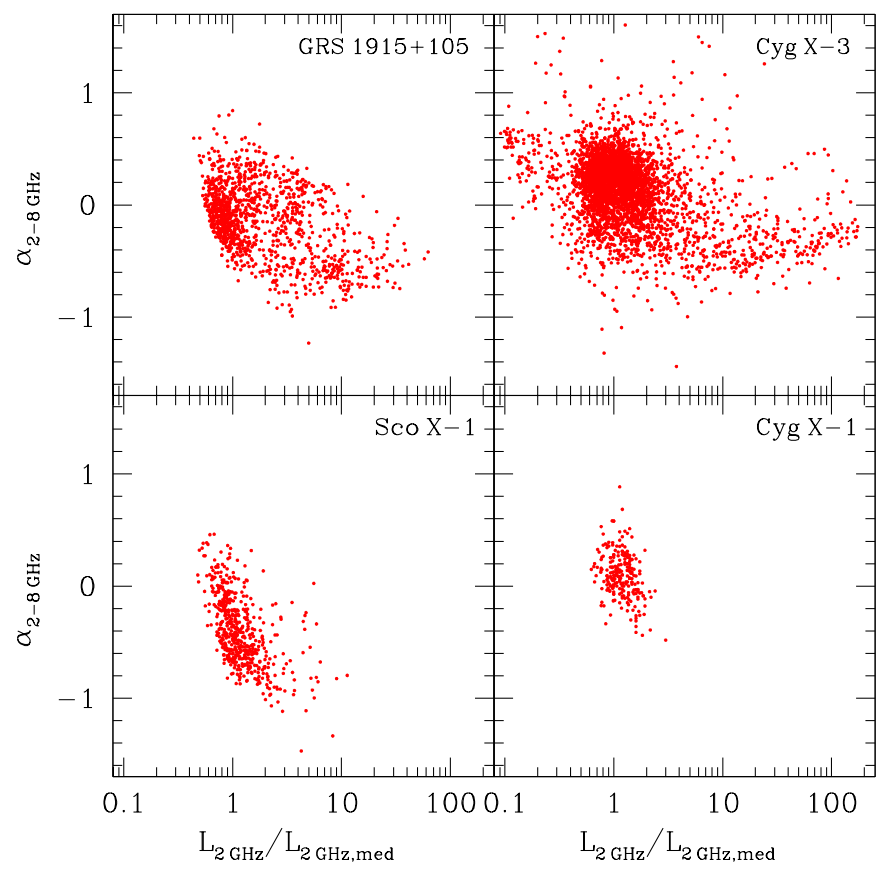

Figure 2: Radio spectral index $\alpha_{2-8 \mathrm{~Hz}}$ as a function of the $2.25 \mathrm{GHz}$ radio luminosity in units of its median value. Each data point refers to the average over one day; only data points with $1-\sigma$ uncertainties on $\alpha_{2-8 \mathrm{~Hz}}$ smaller than 0.25 are plotted.

spent by a microquasar in the flaring mode can be compared with the fraction of quasar $f_{\mathrm{Q}}$ which are radio-loud. On average we found $f_{\mu \mathrm{Q}} \sim 10 \%$, and Ivezić et al. ([3]) report $f_{\mathrm{Q}} \sim 8 \%$ for the Sloan Digital Sky Survey quasars.

The flaring emission has associated jets with relatively large bulk Lorentz factors (e.g. [4]) and is typically characterised by a steeper radio spectrum (see Fig. 2) than the coupled emission, so we speculate that in AGN the flaring mode may be the one responsible for producing radio lobes, while the coupled mode produces only core emission. Thus, different pieces of information suggest a parallel between microquasars in the flaring mode and FR I or FR II sources, and between microquasars in non-flaring states (either low/hard or high/soft) and radio-quiet AGN (with only core radio emission). The absence of extended low-frequency radio lobes associated with radioquiet quasars is not inconsistent with this picture, because the radiative lifetimes of synchrotron particles in lobes are expected to be relatively short ([5]). A consequence of the proposed scenario is that the radio luminosity function of AGN will be dominated by the effects of variability: the data from current radio surveys appear consistent with this hypothesis ([6]).

\section{References}

[1] C. Nipoti, K.M. Blundell, J. Binney, Radio-loud flares from microquasars and radio-loudness of quasars (2005) MNRAS 361633

[2] S. Heinz, Radio lobe dynamics and the environment of microquasars (2002) A\&A 388 L40 
[3] Ž. Ivezić, et al., Optical and Radio Properties of Extragalactic Sources Observed by the FIRST Survey and the Sloan Digital Sky Survey (2002) AJ 1242364

[4] J.C.A. Miller-Jones, K.M. Blundell , M.P. Rupen, A.J. Mioduszewski, P. Duffy, A.J. Beasley, Time-sequenced Multi-Radio Frequency Observations of Cygnus X-3 in Flare (2004) ApJ 600368

[5] K.M. Blundell, S. Rawlings, The spectra and energies of classical double radio lobes (2000) AJ 119 1111

[6] C. Nipoti, J. Binney, Time variability of active galactic nuclei and heating of cooling flows (2005) MNRAS 361428 